\title{
On the minimization of task switch costs following long-term training
}

\author{
Marian E. Berryhill and Howard C. Hughes \\ Dartmouth College, Hanover, New Hampshire
}

\begin{abstract}
Flexible, context-dependent linkages between stimulus and response are fundamental to adaptive behavior. In the present article, we evaluate the limits of this flexibility by exploring the asymptotic efficiency of people's ability to switch between two different sensorimotor mappings. Two stimulus-response (S-R) mappings were learned, either both on the same hand (unimanual condition) or one mapping per hand (intermanual condition). The S-R mappings presented bivalent stimuli and employed the same response keys. A novel training regimen successfully reduced task-switch costs to approximately $20 \mathrm{msec}$, suggesting that residual switch costs cannot be eliminated. These costs cannot be entirely attributed to the cognitive control process of taskset reconfiguration, because they are observed over cued switch intervals of several seconds. Two additional issues in motor learning were addressed: the single or dual loci of manual motor control and the coordinate system of task representation. First, the results favored the notion of independent controllers for each hand instead of a single dominant controller, since intermanual performance was superior to unimanual performance. Second, a transfer task tested internal (egocentric) and external (allocentric) coordinate systems. Transfer was more effective using the external coordinate system, suggesting that the S-R mappings reflected the association between the bivalent stimuli and external goals (i.e., the individual keys) rather than the concrete pattern of muscle contractions (i.e., the finger pressing the key). Finally, retention tests revealed that these learned S-R associations were remarkably durable, since no decrements in fluent task-switching performance were observed after 10 months without practice.
\end{abstract}

A hallmark of human behavior is the ability to respond appropriately to dynamic changes in the environment. For example, a skilled pianist makes seamless transitions between key signatures, whereas a musician of lesser ability persists in playing the notes corresponding to the previous scale (von Kries, 1895, cited in Woodworth $\&$ Schlosberg, 1954). From the perspective of human information processing, the performance of highly skilled pianists suggests that, with sufficient practice, one stimulus-response (S-R) mapping can be replaced with another without any decrement in performance. However, beginning with the seminal studies by Jersild (1927), in investigations of performance on different tasks in succession-task switching-interference effects have been consistently observed whenever switches occur (reviewed in Monsell, 2003). One challenge in developing a theoretical account of task switching is to simultaneously account for the ability of people to exercise executive control operations to endogenously drive a task switch while explaining the performance costs when people implement the task switch.

Switch costs arise from multiple possible sources. One influential view of task switching is to consider the human operator as a finite-state machine. Accordingly, a control signal determines the state of the operator, and each discrete state selects the set of task-appropriate S-R connec- tions (e.g., de Jong, 1995; Meiran, 1996, 2000a, 2000b; Rubinstein, Meyer, \& Evans, 2001). In such a finite state, only the selected task is represented, and there is no trace of an unselected task. Thus, there should be no intertask interference once the system is selected and instantiated. Switch costs could only result in the interval between a new control signal and the subsequent transition to that state. As pointed out by Allport and Wylie (2000), this view suggests that switch costs may occur immediately following a task switch but not once the system is appropriately reconfigured. This view of task switching predicts that all switch costs - even for the trial immediately following a task switch - should be completely eliminated if the appropriate task settings are configured before a response is required. However, advance task cuing reduces - but does not completely eliminate - task-switch costs (Arrington, Logan, \& Schneider, 2007; Jost, Mayr, \& Rösler, 2008; Mayr \& Kliegl, 2003; reviewed in Monsell, 2003; Pashler, 2000 ), suggesting that the finite-state machine hypothesis is insufficient to explain switch costs in general.

What produces these residual switch costs? Residual costs are not attributable to an endogenous control process of task-set configuration, because they persist regardless of the preparation time (Arrington \& Logan, 2004). Use of newly selected S-R connections improves their efficiency, as is evidenced by performance benefits on nonswitch tri-

M. E. Berryhill, berryhil@psych.upenn.edu 
als. This is consistent with the well-known finding that, even in a single-task situation, repeatedly activating the same S-R pathway facilitates response times (RTs; e.g., Theios, 1975). Allport and colleagues (Allport, Styles, \& Hsieh, 1994; Allport \& Wylie, 2000; Wylie \& Allport, 2000 ) suggested that there is long-term facilitation of S-R pathways associated with the unselected task, accompanied by long-term suppression of the S-R pathways of the selected task, despite the best efforts of endogenous control operations to reconfigure the task settings appropriately. They termed the combination of persistent activation of unwanted and suppression of wanted $\mathrm{S}-\mathrm{R}$ pathways task-set inertia. Task-set inertia leads to competing response tendencies when the system is confronted with bivalent stimuli, and thus slows response selection (Hasbroucq, Burle, Akamatsu, Vidal, \& Possamaï, 2001; Osman, Bashore, Coles, Donchin, \& Meyer, 1992; reviewed in Gilbert \& Shallice, 2002; also, cf. the results of Kveraga, Boucher, \& Hughes, 2002, with those of Nelson $\&$ Hughes, 2007).

Switching costs that persist over many trials can also result if the asymptotic strength of the S-R connections differs under single- and dual-S-R-task testing sessions. These long-term effects of the sequential performance of two tasks, relative to single-task performance, have been called the mixing cost (Monsell, 2003; Pashler, 2000). On the basis of this brief overview of the task-switching literature, it appears that the facility of task switching rarely - if ever - approximates the efficiency expected of a finite-state system. Yet, the question remains: How can empirical studies identifying multiple sources of switch costs and interference be reconciled with the astonishing skills of Rachmaninoff, Horowitz, or Monk?

In the present study, a novel training regimen was adopted, with the goal of minimizing all varieties of switching costs as much as possible. In a long-term training period of 5 months, the switch rate between two bivalent S-R mappings steadily increased. Initially, switches between the two S-R mappings were based on attainment of asymptotic performance criteria. Subsequently, switches alternated daily between mappings, and, eventually, switches occurred unpredictably within a session. Within the context of this training regimen, we examined five related experimental questions. First, we investigated whether performance of a second mapping can equal performance on the first mapping; that is, can retroactive and proactive interference (i.e., mixing costs; Monsell, 2003) be eliminated? Second, to what extent can the effects of task-set reconfiguration and other residual costs be reduced? Third, with regard to the issue of shared versus independent controllers for each hand, we asked whether switching between S-R mappings was more efficient under intermanual conditions (one mapping per hand) than under unimanual conditions (both mappings on one hand). Fourth, we examined the facility of task transfer from one hand to the other hand to examine the nature of the $\mathrm{S}-\mathrm{R}$ connections learned during training. This also permitted an evaluation of whether transfer is superior when using an external or internal coordinate system. Fi- nally, we assessed the long-term retention of these learned $\mathrm{S}-\mathrm{R}$ connections after a period with no task practice.

The present results indicate that the performance of two S-R mappings can equal performance of a single $S-R$ mapping, suggesting that mixing costs can be eliminated. We found that switch costs on randomly cued task switches were reduced to an average of less than $20 \mathrm{msec}$, suggesting a remarkably facile degree of cognitive control associated with task-set reconfiguration. Intermanual performance was superior to unimanual performance. This finding supports the traditional view of independent controllers coordinating manual motor control. Finally, with regard to the nature of the $\mathrm{S}-\mathrm{R}$ associations learned under this training regimen, superior performance was observed when the transfer task maintained the external coordinate system rather than internal coordinates. This suggests that the abstract task goal is preserved more readily than the specific pattern of muscle contractions. Task-switching retention was tested after 10 months without practice, and, remarkably, no change in performance was observed. These results indicate that multiple S-R mappings can be learned and maintained at the same level of proficiency over surprisingly long temporal intervals, even though switch costs usually associated with endogenous processes such as taskset reconfiguration cannot be completely avoided.

\section{METHOD}

\section{Subjects}

Twenty right-handed subjects ( 10 men, 10 women, ages $17-42$ years) completed Phases $1-4$ of behavioral training (the training phases are described below). In the retention test (Phase 7), 12 of the original 20 subjects participated -7 from the unimanual group and 5 from the intermanual group. Subjects received $\$ 3$ per session unless they were participating in task-switching training phases, for which they received \$5. The Dartmouth College Committee for the Protection of Human Subjects approved all experimental protocols.

\section{Apparatus}

Stimuli were displayed on a 19-in. CRT monitor at a viewing distance of $57 \mathrm{~cm}$. Subjects' heads were stabilized in a chinrest. Responses were keypresses on a standard keyboard modified so that keypresses were registered as an analog input to a National Instruments data acquisition board. Stimulus presentation and timing were controlled by a generic PC running DOS, which yoked stimulus presentation to the vertical refresh rate of the CRT monitor. RTs and response accuracy were collected and stored to disk for offline analysis using MATLAB 6.5 (MathWorks, Natick, MA).

\section{S-R Mappings}

The stimuli were four circular annuli of $1^{\circ}$ diameter positioned at the corners of an imaginary square at an eccentricity of $8^{\circ}$. A fifth circular annulus of $1^{\circ}$ was placed at the center of the display and served as the fixation point. A red or green border around the five annuli cued the mappings. The intensities of the borders were approximately equiluminant, as determined by heterochromatic flicker photometry. The target luminance was $27 \mathrm{~cd} / \mathrm{m}^{2}$ over a dark background of $3.9 \mathrm{~cd} / \mathrm{m}^{2}$. Each of the four responses was associated with an individual finger (excluding the thumb) and was mapped onto one of the four visual stimuli. There were two different S-R mappings that were inversions of each other. In one mapping, the index and ring fingers responded to the upper two annuli, and the middle and small fingers responded to the lower two annuli. In the second mapping, the index and ring fingers responded to the lower 
Map 1
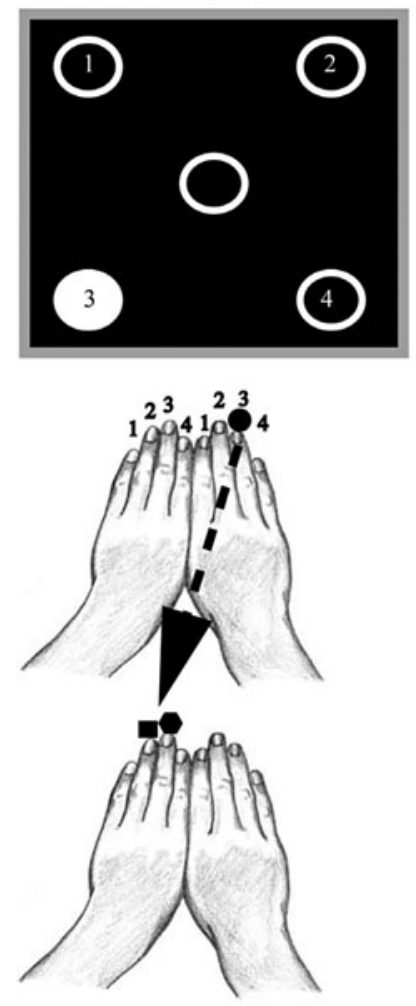

Map 2
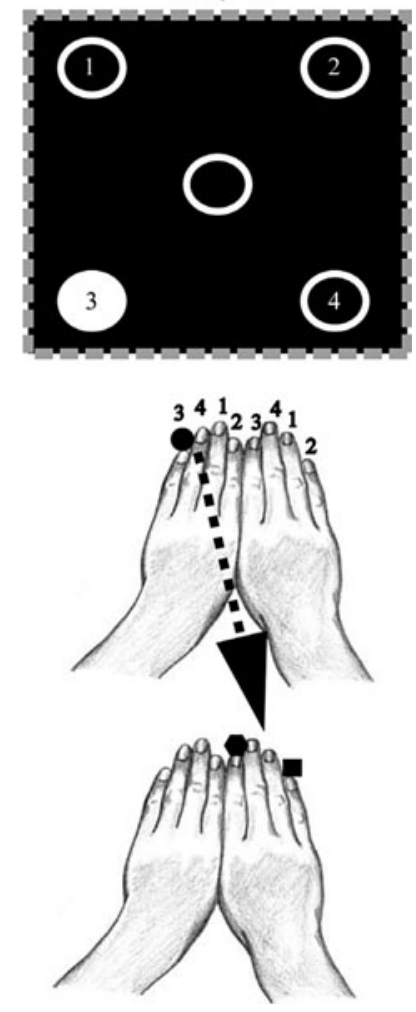

Figure 1. Stimulus-response mappings and transfer schematic. The numbers denote the correspondence between the stimuli and the correct responses. For example, for Map 1, the filled annulus (Number 3) corresponds to a keypress from the right ring finger, as is noted by the solid circle. For the Map 1 task, the correct answer would be a keypress using the middle finger when the task is performed by the left hand and the ring finger when performed by the right. The right column shows Map 2, for which the correct response to Number 3 is a keypress using the small finger when performed by the left hand and the pointer finger when performed by the right hand. The mappings were indicated by two different border colors-red and green, shown here as a solid and a dashed border, respectively. In the example on the left, the trained answer was the right ring finger. For the internal transfer task (marked by a square), the finger pressing the key is maintained, thus the correct answer is a keypress with the left ring finger. For the external transfer condition (marked by a hexagon), the key being pressed is maintained, thus the correct answer is the second finger from the right - the middle finger. Note that the numbers were never visible to the subjects and are shown here for illustration purposes only.

two annuli, and the middle and small fingers responded to the upper two annuli (see Figure 1). In the Results sections, each reference to Map 1 or Map 2 collapses across the specific mapping and refers to the first or the second S-R mapping that the particular subject experienced.

\section{Trial Design}

Each trial began with the onset of a colored border indicating the $\mathrm{S}-\mathrm{R}$ mapping that would be in effect for that trial. The border appeared for an interval of 500-2,500 msec, which gave the subject a variable interval over which to implement their task-set configuration. One of the four peripheral annuli was then illuminated to indicate that it was the target and remained illuminated until a response was recorded. When the response was correct, the next trial began. When an incorrect response was made, the target annulus blinked twice to indicate the mistake. Although response accuracy was indicated to the subject, no feedback was provided regarding the RTs. Each trial lasted approximately $3,000 \mathrm{msec}$ and was followed by a 1,000-msec intertrial interval. Subjects were instructed to emphasize accuracy and speed. Subjects performed experimental sessions of 200 trials. Experimental sessions occurred during successive days during the work week; there was no testing on the weekends or during occasional scheduling conflicts.

\section{Training Paradigm}

The principal difference between this and previous investigations of task switching is the manner and duration of the applied training regimen. The following section describes this training procedure.

Session types. The switch rate between S-R mappings increased in frequency through a series of training phases described below (see Table 1 for a summary).

Phase 1: Acquisition of single mappings and full session alternation. This training phase was characterized by performance of only one S-R mapping during each experimental session. Subjects began the experiment by first learning one of the two alternative S-R mappings. This was counterbalanced across subjects. Subjects continued performing the same mapping until they reached criterion performance. Criterion was reached when the mean correct RT remained 
Table 1

Training Summary, Indicating the Number of Sessions and the Switch Rule for Each Training Phase

\begin{tabular}{lrl}
\hline Training Phase & $\begin{array}{c}\text { Number of } \\
\text { Sessions }\end{array}$ & \multicolumn{1}{c}{ Switch Rule } \\
\hline 1. Full sessions & $32-37$ & $\begin{array}{l}\text { 4 Switches: 3 days within 40 msec (RT) and 5\% (accuracy) } \\
\text { 9 Switches: Daily } \\
\text { 2. Long blocks }\end{array}$ \\
3. Short blocks & 10 & $\begin{array}{l}\text { 40 trials } \\
\text { 10 trials }\end{array}$ \\
4. Alternating & 6 & 1 trial \\
5. Random & $2-9$ & Random \\
6. Transfer & 1 & 50 trials \\
7. Retention & 1 & 1st 100 trials: 1 block Map 1, 1 block Map 2 \\
& & 2nd 100 trials: Random \\
\hline
\end{tabular}

Note-RT, response time

within $40 \mathrm{msec}$ and accuracy remained within a 5\% range across three consecutive testing sessions. Only then did subjects learn the other S-R mapping, which they then performed until they reestablished criterion performance. Subjects then alternated between the two S-R mappings until they had achieved the criterion level of performance four times on each mapping.

In the remaining Phase 1 sessions, the S-R mappings alternated with one mapping rule in effect for each experimental session. The total number of sessions varied for each subject, since individuals reached criterion after different numbers of sessions. There was a total of seven periods for each mapping.

Phase 2: Long block switching. During this and all subsequent phases, subjects performed both mappings during each session. The mapping that was performed first was alternated over daily sessions, and the tasks were switched after blocks of 40 trials. There were 10 Phase 2 sessions.

Phase 3: Short block switching. During this training phase, S-R mappings alternated after blocks of 10 trials. As in Phase 2, the mapping performed first alternated over daily sessions. There were six Phase 3 sessions.

Phase 4: Alternating trials. S-R mappings were switched after each trial. There were six Phase 4 sessions.

Phase 5: Random switching. S-R mappings switched randomly and with equal likelihood from trial to trial. Thus, the numbers of trials per mapping were equal. There were between two and nine sessions. This number varied because subjects performed this phase until they participated in a neuroimaging session (data not included)

Phase 6: Transfer. Subjects performed one block of 100 trials per $\mathrm{S}-\mathrm{R}$ mapping using the untrained hand. Two types of intermanual transfer were tested: external, in which the correct response key was preserved, whereas the effector changed, and internal, in which the correct effector was preserved but the key pressed changed. Transfer was tested in a between-subjects manner, such that each subject only conducted one transfer task. This avoided concerns of interference between transfer tasks that might have resulted if the same subject had performed both types of transfer. Transfer assignment is shown in the lower panel of Figure 1.

Phase 7: Long-term retention. Following a period of at least 10 months with no practice, 12 subjects returned for a retention test. Subjects performed a block of 50 trials per mapping and then 100 trials under random switching conditions. Subjects were not told at the end of training that there would be a retention test in the future.

\section{RESULTS}

\section{Phase 1: Single Mappings}

Rate of unimanual or intermanual mapping acquisition. Phase 1 revealed the time course of S-R map learning. In the Phase 1 sessions, we measured the rate of achieving criterion performance for each $\mathrm{S}-\mathrm{R}$ mapping- whether practiced unimanually or intermanually - to evaluate whether the numbers of sessions to criterion for the unimanual and intermanual groups differed. There was no difference in the numbers of sessions to initial criterion, as measured by a two-tailed $t$ test comparing unimanual (mean number of sessions to criterion $=8.05$ ) and intermanual $(M=8.00)$ groups $[t(18)=1.46, p=.16]$. These results indicate that the early learning of two S-R mappings on one hand did not impose additional interference when compared with learning the mappings on different hands.

Comparing Map 1 and 2 proficiency. We next evaluated whether performance on the second mapping was equivalent to the level of performance on the first mapping. The data from the eight criterion points of Phase 1 (four per S-R mapping) were chosen. These data points reflect performance when subjects maintained performance across 3 days, which should demonstrate a stable level of proficiency. A repeated measures MANOVA was conducted, comparing performance at criterion (RT, accuracy), mapping (Map 1, Map 2), and criterion point per mapping (1-4) separately for the unimanual and intermanual groups. The unimanual group performed the first mapping more rapidly than the second mapping but equally as accurately [RT, $F(1,9)=5.09, p=.05$; accuracy, $F(1,9)=1.26, p=.29$ ], although the difference in means along both dimensions was numerically small (mean RT, Map $1=402.5 \mathrm{msec}$, Map $2=422.7 \mathrm{msec}$; mean accuracy, Map $1=96.6 \%$, Map $2=95.9 \%$ ). The main effect of criterion point was also significant [RT, $F(3,27)=15.54, p<.001 ;$ accuracy, $F(3,27)<1.0$, n.s.], such that performance of the first criterion level was significantly slower than later criterion levels (all $p$ s $<.03$ ); the second through fourth criterion levels were not statistically different from each other (all $p \mathrm{~s}>.07$ ). The interaction of map and criterion level did not reach significance for RT or accuracy [RT, $F(3,27)<1$, n.s.; accuracy, $F(3,27)=2.01, p=.14]$. In contrast, the intermanual group showed no significant difference in RT $[F(1,9)<1$, n.s.] but did reveal a significant main effect of accuracy $[F(1,9)=8.74, p=.02]$ between mappings. Again, the numerical differences between values are small (RT, Map $1=418.4 \mathrm{msec}$, Map $2=421.4 \mathrm{msec}$; accuracy, Map $1=96.5 \%$, Map $2=95.7 \%$ ). The interaction of map and criterion level did not reach significance for RT or accuracy [RT and accuracy, $F(3,27)<1$, n.s.]. The main ef- 

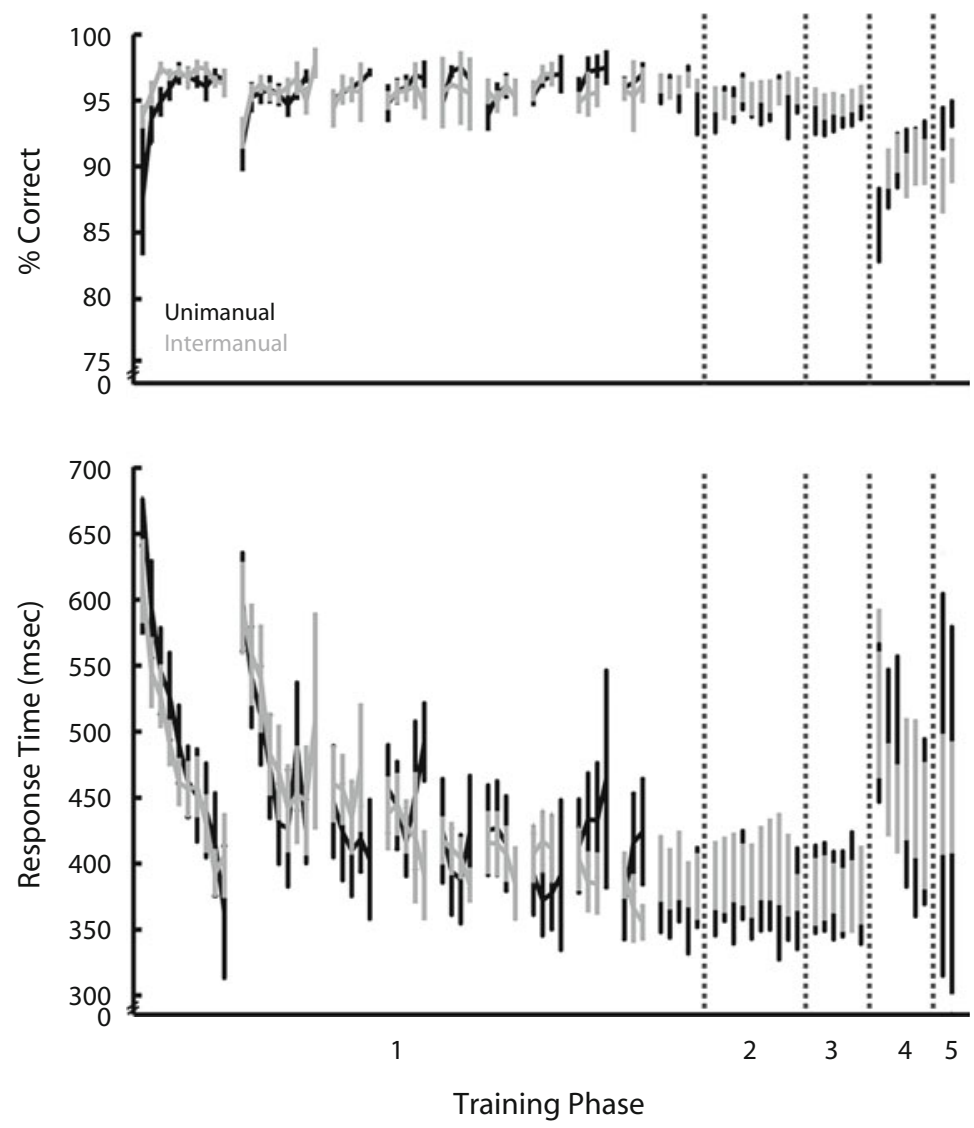

Figure 2. Behavioral response time and accuracy data for the unimanual and intermanual conditions for the training Phases 1-5: (1) full sessions, (2) long blocks, (3) short blocks, (4) alternating, and (5) random. The data are collapsed across map color and hand. The error bars represent the standard errors of the means.

fect of criterion point reached significance $[F(3,27)=5.2$, $p=.01]$, but no pairwise contrasts reached significance.

In a second analysis, performance levels from the final 2 days of Phase 1 were compared, and no significant differences were found in RT or accuracy for either the unimanual or intermanual groups (all $p \mathrm{~s}>.1$ ). These results suggest that, after the initial criterion points for each mapping, there was no difference in performance between the two mappings for either group.

Learning curves: Maps 1 and 2. The most dramatic improvements in performance reflect the learning during Phase 1. In order to compare the rates of learning between the two mappings, we compared the best-fitting functions for the RT and accuracy data. Power law functions that best fit the first series of sessions for the unimanual [Map 1, $y=.72 x^{-.27}, F(1,94)=77.52, p<.0001$, adjusted $r^{2}=$ .45 ; Map 2, $y=.60 x^{-.18}, F(1,62)=22.76, p<.0001$, adjusted $r^{2}=.26$ ] and intermanual [Map 1, $y=.62 x^{-.18}$, $F(1,82)=72.29, p<.0001$, adjusted $r^{2}=.26$; Map 2, $y=.60 x^{-.14}, F(1,74)=16.81, p=.0001$, adjusted $r^{2}=$ .17] groups were not different. There were no significant differences between groups (all $p \mathrm{~s}>.09$ ). These findings indicate that both groups learned each mapping following a similar learning curve. See Figure 2.

\section{Phases 2 and 3: Long and Short Block Performance}

The results from the long and short block sessions show asymptotic performance for both mappings and both groups. There was no statistical difference between the unimanual and intermanual groups for any of the long or short block time points for measures of RT or accuracy (all $p \mathrm{~s}>$.19). The final training phases show no statistically significant differences in performance between groups when compared using two-tailed independent $t$ tests (all $p s>.1)$. These data indicate that both mappings and both groups achieved the same level of proficiency during the extended training period.

Patterns of errors. To further compare interference effects between the two mappings, we examined the patterns of errors in the unimanual and intermanual groups. The errors of primary interest were those that corresponded to the correct answer for the mapping that was not activated, termed the correct other mapping errors. The proportion of each error type is shown in Figure 3. The second type of error corresponded to pressing one of the other two fingers that did not correspond to the correct answer for the incorrect mapping; these are termed neighbor errors. Errors early in training were characterized as neighbor er- 


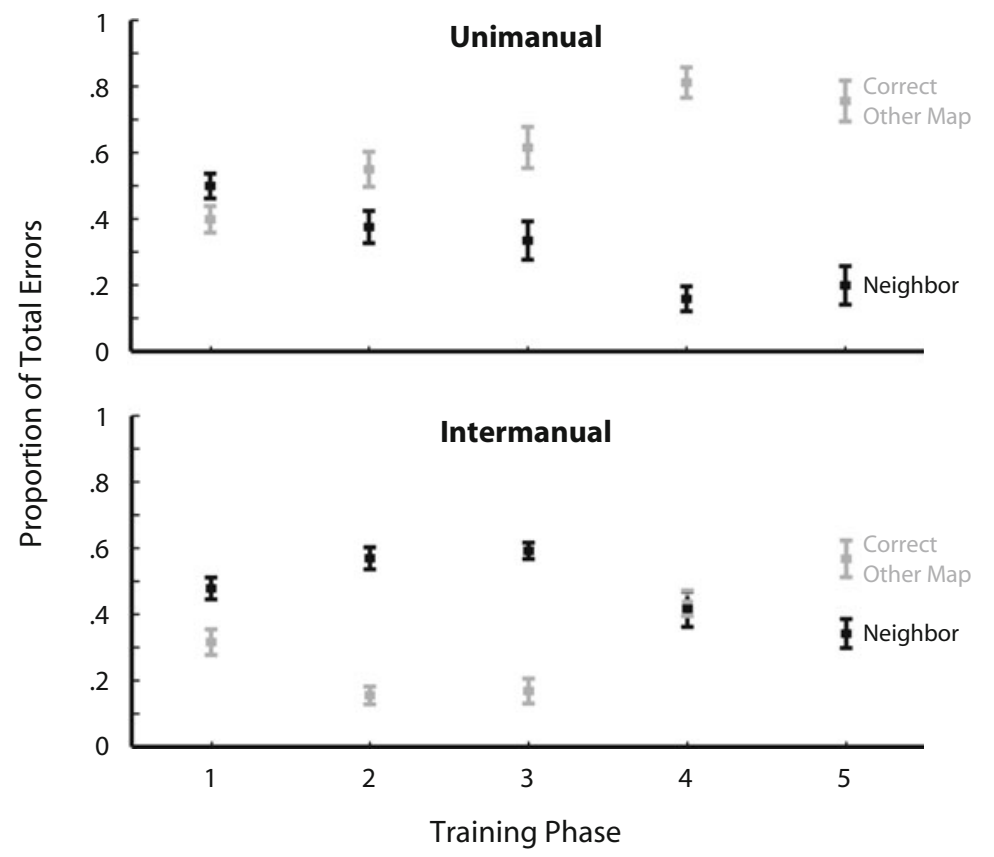

Figure 3. Error data for unimanual (top) and intermanual (bottom) conditions across Phases 1-5. Two classes of errors are noted: neighbor errors, in which a finger bordering the correct response finger responded (i.e., the middle finger instead of the pointer finger made the response) and correct other errors, in which the correct response for the other mapping was provided. The $y$-axis represents the proportion of total errors of each type that were committed during each training phase. Error bars represent the standard errors of the means.

rors, and, over the course of training, the errors patterned such that people were more likely to press the correct response for the incorrect mapping. There was a small proportion of a third error type - a response by the fourth finger. There were few of these responses, and they are not of theoretical interest and are not included in the analyses. In order to assess whether there was a differential shift in error types between the two groups over time, these data were compared in an ANOVA with the within-subjects factors of phase (1-5) and error type (neighbor, correct other mapping) and between-subjects factor of group (unimanual, intermanual). There was a significant twoway interaction between phase and error type $[F(4,72)=$ $38.3, p<.001]$, indicating that both groups demonstrated a significant shift in error types across training phases. However, the three-way interaction between group, phase, and error type $[F(4,72)=10.8, p<.001]$ confirmed that the unimanual group committed more correct other mapping errors later in training. This finding indicates that there was stronger interference between the two mappings in the unimanual group.

In a second analysis, we compared errors occurring during the map switch and nonswitch trials during the random switching phase (Phase 5). The majority of the errors during this phase $(647 / 996=.65)$ were performed during the unpredictable switch trials. This proportion of switch errors was significantly different from the expected value of $.5[t(19)=-4.61, p=.0002]$, indicating that more errors occurred during switch trials than during nonswitch trials. These data demonstrate a second between-groups dissociation. The unimanual group committed a disproportionate number of switch errors (mean switch errors $=.72$ ) when compared with the intermanual group (mean switch errors $=.57$ ). After removing two outliers, which were farther than two standard deviations from the mean, the difference between the unimanual and intermanual proportions of switch errors reached statistical significance $[t(18)=$ $-2.75, p=.013]$, such that the proportion of switch errors was significantly higher in the unimanual group.

\section{Phase 5: Costs of Random Switching}

Switch costs were examined to assess whether they could be eliminated or reduced by extensive training or by intermanual training. Switch costs were investigated using the data from the random switching phase (Phase 5) because these sessions occurred after extensive training and included switch and nonswitch trials. Switch costs were calculated by subtracting the mean nonswitch RT from the mean switch RT.

Switch costs were first assessed as a function of the border-cue duration. This cue signaled which mapping would be in effect when the target appeared. For all correct trials, cue duration was binned in 100-msec segments from 900 to 2,400 msec. Switch costs were determined for 

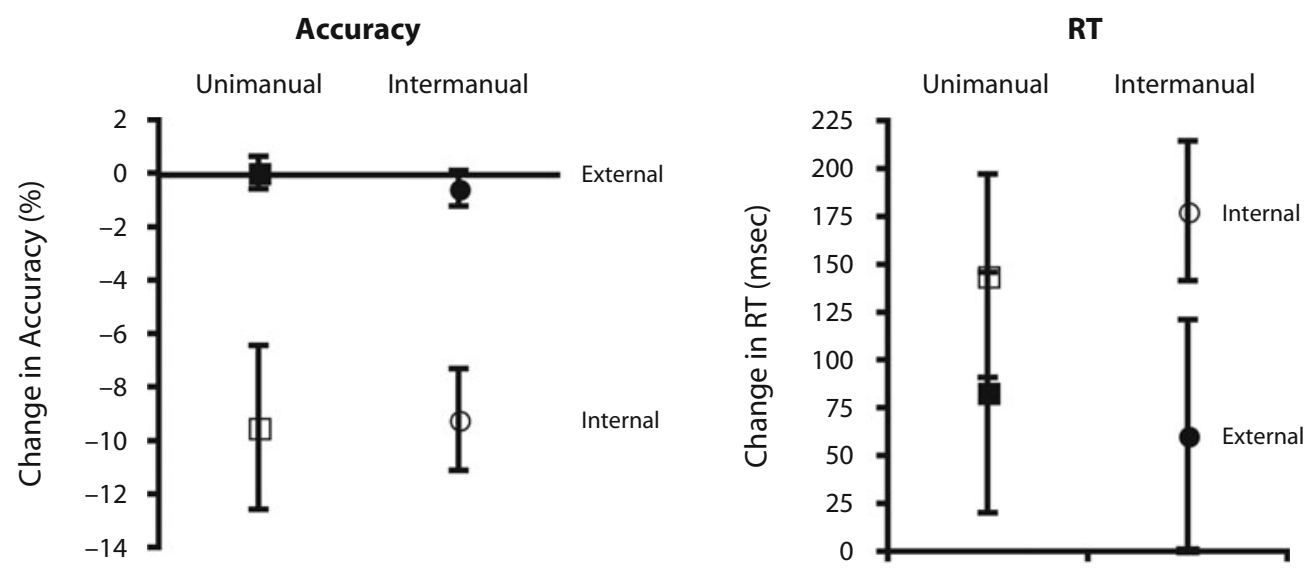

Figure 4. Transfer data from the unimanual and intermanual groups for internal and external transfer conditions. The difference scores were calculated by subtracting the transfer performance from the long block performance. The mean and standard error of the mean were then determined for each group. The left panel presents the change in accuracy from the trained condition; any data below 0 reflect poorer performance than that during training. The right panel presents the change in response time (RT); any data above 0 reflect slower performance than that during training.

each subject for each bin and were subjected to repeated measures ANOVAs with group as a between-subjects factor. Neither the main effect of delay or group nor the interaction of group and delay achieved statistical significance (all $F \mathrm{~s}<1$, all $p \mathrm{~s}>.3$ ). These data suggest that small switch costs were not eliminated with longer border-cue durations.

Since there was no significant difference in switch costs as a function of cue duration, data were collapsed across cue durations in subsequent analyses. One outlying data point was removed from each group because each was farther than two standard deviations from the mean. The unimanual outlier demonstrated extremely high switch costs (154 msec), whereas the intermanual outlier showed great facilitation for switch trials $(95 \mathrm{msec})$. The remaining data revealed comparable switch costs for each group (unimanual mean $=18.10 \mathrm{msec}$, intermanual mean $=$ $12.11 \mathrm{msec}$ ), which did not demonstrate a significant difference between groups $[t(16)=0.58, p=.57]$. This analysis suggests that the time to switch $\mathrm{S}-\mathrm{R}$ representations does not differ when mappings are performed by a single hand or by two hands.

\section{Phase 6: Task Transfer}

In order to evaluate the dominant coordinate system of task transfer, we evaluated performance on internal and external transfer tasks. Difference scores between performance using the standard mapping (Phase 3 short block performance, for similarity to transfer design) and transfer performance were calculated (see Figure 4). External coordinate transfer modestly affected accuracy (mean change in accuracy, unimanual $=+0.02 \%$, intermanual $=-0.57 \%$ ) and RT (mean change in RT, unimanual $=+83.0 \mathrm{msec}$, intermanual $=+61.0 \mathrm{msec})$. In contrast, the internal coordinate transfer produced greater impairment in terms of accuracy (mean change in accu- racy, unimanual $=-9.52 \%$, intermanual $=-9.30 \%)$ and RT (mean cost in RT, unimanual $=+144 \mathrm{msec}$, intermanual $=+178 \mathrm{msec}$ ). These measures were subjected to a MANOVA comparing transfer (external, internal) with group (unimanual, intermanual). There was a significant main effect of transfer type $[F(2,15)=13.78, p<.001]$ but no main effect of group $[F(2,15)=0.06, p=.95]$ or interaction $[F(2,15)=0.14, p=.87]$. The significant effect of transfer type was dominated by the significantly lower accuracy in the internal transfer group. The results from the transfer task indicate that external coordinate transfer was performed with greater facility than was internal coordinate transfer.

\section{Phase 7: Long-Term Retention}

Subjects performed 56-68 training sessions over approximately 5 months. The opportunity remained to evaluate the long-term retention of the two mappings after at least 10 months without practice. The RT and accuracy data from the block session were compared with performance during the short block training (Phase 3), because the short block phase reflected similar testing conditions (see Table 2).

There was one outlier from the unimanual group who showed a 219-msec slowing for the first mapping and a 42-msec improvement for the second mapping. This individual was the subject with the slowest RTs throughout training. Excluding this subject's data revealed a negligible RT difference of $1.7 \mathrm{msec}$. Analysis by MANOVA comparing experimental period (training, retention) with measure (Phase 3: RT, accuracy) revealed no significant main effects or interactions (all $p \mathrm{~s}>.40$ ).

In a second analysis, we compared performance from the immediately preceding training phase - the random switching phase (Phase 5). Again, the previously mentioned outlier subject showed a large $(242-\mathrm{msec})$ slow- 
Table 2

Retention Test Performance

\begin{tabular}{|c|c|c|c|c|c|}
\hline \multirow[b]{3}{*}{ Subject } & \multirow[b]{3}{*}{ Measure } & \multicolumn{4}{|c|}{ Difference Score } \\
\hline & & \multicolumn{2}{|c|}{ Block } & \multicolumn{2}{|c|}{ Random } \\
\hline & & $M$ & SEM & $M$ & SEM \\
\hline Unimanual & $\begin{array}{l}\text { RT } \\
\text { Accuracy }\end{array}$ & $\begin{array}{r}-14.13 \\
+0.29\end{array}$ & $\begin{array}{r}14.21 \\
1.75\end{array}$ & $\begin{array}{r}-32.73 \\
+3.08\end{array}$ & $\begin{array}{r}18.64 \\
2.16\end{array}$ \\
\hline Intermanual & $\begin{array}{l}\text { RT } \\
\text { Accuracy }\end{array}$ & $\begin{array}{l}-3.61 \\
-0.88\end{array}$ & $\begin{array}{r}12.61 \\
1.07\end{array}$ & $\begin{array}{l}+0.73 \\
-3.11\end{array}$ & $\begin{array}{r}15.92 \\
1.98\end{array}$ \\
\hline
\end{tabular}

Note-Difference Score, changes in response time (RT, in milliseconds) and accuracy (\%) between the block and random retention tasks (trained score - retention score). Positive numbers indicate faster or more accurate performance on the retention task; negative numbers indicate slower or less accurate performance.

ing of RTs for the first mapping. Excluding this outlier revealed a slowing of 15.3 msec. A MANOVA comparing retention period (training, retention) with measure (Phase 5: RT, accuracy) including these data replicated the above findings, and no significant main effects or interactions were found (all $p \mathrm{~s}>.09$ ).

These data indicate that retention for the two groups was equivalent and that performance after retention was equal to performance during training. This suggests that both mappings were successfully consolidated and that these representations were rendered immutable, at least over a 10-month period without practice.

\section{DISCUSSION}

In the present study, a novel long-term training paradigm was used to minimize sources of task-switch costs in order to assess the asymptotic flexibility of switching between two sets of S-R connections, the coordinate structure of the connections formed, the degree to which performance on each task interferes with the other's proficiency, and the endurance of task consolidation. Five months of training produced over 280,000 trials in a simple visuomotor task using bivalent stimuli to establish two equally difficult $\mathrm{S}-\mathrm{R}$ mappings. Two training groups were compared: the unimanual group, who learned both $\mathrm{S}-\mathrm{R}$ mappings with the right hand, and the intermanual group, who learned each mapping on a different hand. A series of training phases imposed steadily increasing demands on response flexibility by accelerating the rate of map switching from one mapping per session (Phase 1) to sessions of randomized map switching (Phase 5). Following this extensive training, remarkably modest switch costs of less than 20 msec remained. The comparison of the unimanual and intermanual groups revealed that subjects were able to acquire and maintain equivalent proficiency with both mappings. Between-groups comparisons demonstrated that the intermanual group was more resistant to interference between mappings than was the unimanual group and was therefore superior. In order to assess the preferred coordinate system of the stored task, we compared task transfer (Phase 6) to the untrained hand when we applied internal coordinates with that when we applied external coordinates. With respect to testing transfer, performance was best preserved by implementing an external coordinate system. This suggests that task representation corresponded to the end goal rather than to a specific motor pattern. However, transfer implementing an internal coordinate system also demonstrated a high degree of accuracy. Finally, proficiency for both mappings was retained equally well by unimanual and intermanual groups even after a 10month period without performing the task (Phase 7). In the following paragraphs, the implications of these findings are addressed.

\section{The Finite-State Machine and Switching Costs}

Prior to this study, there was little empirical evidence that task-switching performance could ever approximate the performance of a finite-state machine. Rather, most studies emphasized multiple sources of interference, including the time taken by executive control processes to reconfigure the system and to enable the new task (Arrington \& Logan, 2004; Kimberg, Aguirre, \& D'Esposito, 2000; Logan \& Bundesen, 2003, 2004; Schneider \& Logan, 2005), interference from previous S-R mappings (Allport et al., 1994; Allport \& Wylie, 2000), and endogenous and exogenous shifting of S-R mapping (Rogers \& Monsell, 1995). Even a finite-state system would need time to reconfigure the task, so some switch costs would likely remain under the conditions in which task switches occur unpredictably - and with little warning. However, even with advanced reconfiguration time, switch costs remain (Arrington et al., 2007; Mayr \& Kliegl, 2003; Meiran, 1996). Impaired performance has been demonstrated when there is a pause in the task that is not accompanied by a task switch, suggesting a resurrection of interference from the competing task (Schneider \& Logan, 2007). These findings confirm that assuming a finite-state machine does not provide sufficient explanatory power.

A second possible source of switch costs was mixing costs - the general performance deficit sometimes observed when multiple tasks are performed (Los, 1996). In the present data, these costs were undetected. On the two tasks, subjects achieved equal asymptotic facilities in the unimanual and intermanual conditions when performed in isolation and when both tasks were performed during a single session. This result suggests that mixing costs can be eliminated or rendered invisible.

We also saw little evidence of task-set inertia or of spontaneous intrusions of the alternate S-R mapping. Although switch costs remain, it is important to emphasize that, if given about $1 \mathrm{sec}$ to reconfigure their task settings, the present subjects had residual switch costs of less than $20 \mathrm{msec}$ ! This suggests that, in the limit, task-switch costs can approximate the predictions of a finite-state machine hypothesis. This remarkable degree of task-switching facility is the primary result of this investigation, and it illustrates how close the asymptotic efficiency of well-learned task switching can approximate a memoryless finite-state system. The results confirm and extend those of earlier work demonstrating that task-switch efficiency can be 
maximized through extensive training (Stoet \& Snyder, 2003, 2007), task familiarity (Rubinstein et al., 2001), high S-R compatibility (e.g., Dassonville, Lewis, Foster, \& Ashe, 1999; Fitts \& Deininger, 1954; Fitts \& Seeger, 1953; Fitts \& Simon, 1952; Kornblum \& Lee, 1995; Proctor, Wang, \& Vu, 2002; Simon \& Rudell, 1967; Simon \& Small, 1969; reviewed in Lien \& Proctor, 2002), and S-R mapping cue (Diedrichsen, Hazeltine, Kennerley, \& Ivry, 2001; Hazeltine, Bunge, Scanlon, \& Gabrieli, 2003; Lien $\&$ Proctor, 2002).

For a host of practical reasons, few studies endeavor to test subjects over the extended time periods used in the present study. However, these results demonstrate that many frequently observed sources of between-task interference can be eliminated if people are given sufficient practice. In the present study, we successfully reduced switch costs to less than $20 \mathrm{msec}$, even though the task switching occurred at random intervals. This is consistent with a recent report in which Stoet and Snyder (2007) collected at least 23,800 trials from each of 4 subjects in a two-response task-switching paradigm. Modest switch costs remained and no significant improvement was observed during the second half of the trials. It is clear that switching costs are not easy to eliminate, but even the present results involved only 5 months of training. Perhaps years of practice can eliminate these costs entirely.

One explanation for the durability of switch costs is that they are due to an indelible degree of top-down interference. It has been shown that well-trained primates exhibit negligible switch costs, whereas humans performing the same task demonstrate greater switch costs (Stoet \& Snyder, 2003). In recent studies, Dreisbach and colleagues (Dreisbach, Goschke, \& Haider, 2007; Dreisbach \& Haider, 2008) investigated whether the underlying cause of this dissociation between species was due to differences in the cognitive representations of the two task sets. To test this hypothesis, Dreisbach et al. taught human subjects eight S-R pairings that could also be conceptualized as two tasks with four possible responses each. Surprisingly, no switch costs were observed until the unnoticed taskset structure was revealed (Dreisbach et al., 2007). In a subsequent study, Dreisbach and Haider suggested that this interference may reflect a beneficial process in which external S-R pairings are suppressed in order to optimize performance on the current task. Their data indicate that representing the S-R mappings as distinct sets is sufficient to introduce switch costs. This cognitive limitation may be rendered beneficial by confining responses to relevant options when performing a task. Such a telescoping process might serve to limit errors and to promote the creation of context dependence - hallmarks of executive function (Miyake et al., 2000).

\section{Intermanual and Unimanual Differences and Motor Control}

In the present study, the comparison between unimanual and intermanual performance provides limited support for the superiority of between-hand performance. Sub- jects in the unimanual group were more likely to make errors that corresponded to the correct answer in the other mapping. This is consistent with some previous findings reporting reduced switch costs when S-R mappings are lateralized across hands (Bradshaw, Nicholls, \& Rogers, 1998; but see Charron, Collin, \& Braun, 1996, and Nefs, Kappers, \& Koenderink, 2005). The finding that interference is not completely eliminated under conditions of intermanual task switching (e.g., Braun, Hess, Burkhardt, Wühle, \& Preissl, 2005; Thut et al., 1996) may be due to interhemispheric connections (Diedrichsen, Hazeltine, Nurss, \& Ivry, 2003), such as the callosal links between the hand representations in the two hemispheres (Wahl et al., 2007).

Greater interference and variability in the unimanual condition suggest that independent motor control guided performance for each hand. In contrast, neuroimaging findings suggest a dominant left supplementary motor area that controls right as well as left motor responses in sequence-learning tasks (e.g., Grafton, Hazeltine, \& Ivry, 1998, 2002). The results of these studies suggest the presence of a single dominant controller located in the left hemisphere. One difference that may explain these different findings is the nature of the tasks. The dominant hemisphere motor controller may be necessary to coordinate bimanual motor sequences, and independent controllers may suffice for simple S-R motor responses, such as those used here.

\section{Coordinate System and Direction of Task Transfer}

In order to investigate the manner of task representation, task transfer was tested under two coordinate systems: internal (egocentric) and external (allocentric). Transfer based on internal coordinates maintains the homological pattern of muscle movement, such that the finger pressing the key remains the same. This corresponds to a concrete, low-level representation in which the responses are reassigned to the corresponding fingers of each hand. Precise interhemispheric connections between homological representations could be the mechanism of transfer based on internal coordinates, and evidence in support of this type of transfer comes from distorted field paradigms (Krakauer, Pine, Ghilardi, \& Ghez, 2000; Malfait, Shiller, \& Ostry, 2002; Shadmehr \& Moussavi, 2000; Shadmehr \& MussaIvaldi, 1994).

In contrast, transfer based on external coordinates preserves the goal state, such that the physical key pressed remains the same. Evidence supporting external coordinate transfer comes from complex motor paradigms (Imamizu \& Shimojo, 1995; Willingham, Wells, Farrell, \& Stemwedel, 2000). Conciliation between these contrasting views comes from dual-layer models of task representation, which propose that each coordinate system corresponds to a different task representation. The external coordinate frame corresponds to the consolidated goal representation found in well-learned tasks. A second, more concrete representation determines the effector-specific motor response corresponding to the internal coordinate frame (Cohen, Pascual- 
Leone, Press, \& Robertson, 2005; Vangheluwe, Wenderoth, \& Swinnen, 2005; Willingham, 1998). Insofar as the present results demonstrated transfer along egocentric or allocentric coordinate systems, our results suggest that both representations were available to our subjects, a finding that is consistent with a dual-layer account.

Subjects were able to transfer performance well using either coordinate system. This finding suggests that both representations exist and are accessible. However, transfer using the external coordinate system was more efficient than the transfer using the internal coordinate system. Performance using the external coordinate system showed preserved accuracy, but only between trained and transfer task performance, and showed only a modest slowing in RT when the external coordinates were preserved. These data provide an indication that the abstract representation dominated the concrete representation. This pattern of findings is consistent with the dual-layer movement representation (Vangheluwe et al., 2005), in which effectorspecific (concrete) and effector-independent (abstract) representations reside concurrently in different cortical locations. Alternatively, these data could be interpreted to indicate that a single, flexible task representation for both mappings was developed (Hazeltine, 2005). This account suggests that the motor responses are conceptualized in terms of the goal.

Some evidence suggests that, although internal coordinates may be directly accessed, external coordinates must first be transformed (Lange, Braun, \& Godde, 2006). Our data suggest that if a transformation step is needed, it can be terminated more quickly than can the process accessing internal coordinate transfer. One possibility is that this task was overlearned to the degree that a concrete representation was no longer needed, and fewer cortical resources were required for task performance. Support for this view comes from neuroimaging studies in which reduced motor activity is observed following training (Floyer-Lea \& Matthews, 2004; Jueptner et al., 1997; Puttemans, Wenderoth, \& Swinnen, 2005; Ungerleider, Doyon, \& Karni, 2002). Further research is needed to determine the time course over which S-R mappings are consolidated and whether this process is consistent across tasks.

An additional issue relating to task transfer deserves mention. Although evidence of task transfer has been found in diverse paradigms (Cunningham \& Welch, 1994; Perez et al., 2007; Sathian \& Zangaladze, 1998; Schulze, Lüders, \& Jäncke, 2002; Teixeira, 2000), there is little consistency concerning its direction. Conflicting reports have observed bidirectional transfer between hands (Morton, Lang, \& Bastian, 2001), unidirectional transfer solely from the dominant to the nondominant limb (Criscimagna-Hemminger, Donchin, Gazzaniga, \& Shadmehr, 2003; Halsband, 1992; Lange, Godde, \& Braun, 2004; Thut et al., 1996; Wang \& Sainburg, 2004), and unidirectional transfer from the nondominant to the dominant limb (Taylor \& Heilman, 1980). The present data reveal successful bidirectional transfer. Given the longitudinal nature of the present task, extensive training may lead to equivalent bidirectional transfer, whereas a lesser degree of training may result in unidirectional transfer. Alternatively, the direction of transfer may be task dependent, and the bivalent stimuli used here may have been too simple or overlearned to reveal asymmetries in transfer performance.

\section{AUTHOR NOTE}

We thank Peter U. Tse, Scott T. Grafton, Terrance Darcey, and John D. Van Horn for their comments and suggestions during the course of these studies. Correspondence concerning this article should be sent to M. E. Berryhill, Center for Cognitive Neuroscience, University of Pennsylvania, 3720 Walnut Street, B-51, Philadelphia, PA 19104 (e-mail: berryhil@, psych.upenn.edu).

Note-Accepted by the previous editorial team, when Thomas H. Carr was Editor.

\section{REFERENCES}

Allport, D. A., Styles, E. A., \& Hsieh, S. (1994). Shifting intentional set: Exploring the dynamic control of tasks. In C. Umiltà \& M. Moscovitch (Eds.), Attention and performance XV: Conscious and nonconscious information processing (pp. 421-452). Cambridge, MA: MIT Press.

Allport, D. A., \& Wylie, G. (2000). Task switching, stimulus-response bindings, and negative priming. In S. Monsell \& J. Driver (Eds.), Control of cognitive processes: Attention and performance XVIII (pp. 3570). Cambridge, MA: MIT Press.

Arrington, C. M., \& Logan, G. D. (2004). The cost of a voluntary task switch. Psychological Science, 15, 610-615.

Arrington, C. M., Logan, G. D., \& Schneider, D. W. (2007). Separating cue encoding from target processing in the explicit task-cuing procedure: Are there "true" task switch effects? Journal of Experimental Psychology: Learning, Memory, \& Cognition, 33, 484-502.

Bradshaw, J. L., Nicholls, M. E., \& Rogers, M. A. (1998). An intermanual advantage for tactual matching. Cortex, 34, 763-770.

Braun, C., Hess, H., Burkhardt, M., Wühle, A., \& Preissl, H. (2005). The right hand knows what the left hand is feeling. Experimental Brain Research, 162, 366-373.

Charron, J. F., Collin, I., \& Braun, C. M. J. (1996). Intermanual transfer of somaesthetic information: A two-point discrimination experiment. Neuropsychologia, 34, 873-877.

Cohen, D. A., Pascual-Leone, A., Press, D. Z., \& Robertson, E. M. (2005). Off-line learning of motor skill memory: A double dissociation of goal and movement. Proceedings of the National Academy of Sciences, 102, 18237-18241.

Criscimagna-Hemminger, S. E., Donchin, O., Gazzaniga, M. S., $\&$ SHADMEHR, R. (2003). Learned dynamics of reaching movements generalize from dominant to nondominant arm. Journal of Neurophysiology, 89, 168-176.

Cunningham, H. A., \& Welch, R. B. (1994). Multiple concurrent visual-motor mappings: Implications for models of adaptation. Journal of Experimental Psychology, 20, 987-999.

Dassonville, P., Lewis, S. M., Foster, H. E., \& Ashe, J. (1999). Choice and stimulus-response compatibility affect duration of response selection. Cognitive Brain Research, 7, 235-240.

DE JONG, R. (1995). Strategical determinants of compatibility effects with task uncertainty. Acta Psychologica, 88, 187-207.

Diedrichsen, J., Hazeltine, E., Kennerley, S., \& Ivry, R. B. (2001). Moving to directly cued locations abolishes spatial interference during bimanual actions. Psychological Science, 12, 493-498.

Diedrichsen, J., Hazeltine, E., Nurss, W. K., \& Ivry, R. B. (2003). The role of the corpus callosum in the coupling of bimanual isometric force pulses. Journal of Neurophysiology, 90, 2409-2418.

Dreisbach, G., GoschKe, T., \& Haider, H. (2007). The role of task rules and stimulus-response mappings in the task switching paradigm. Psychological Research, 71, 383-392.

Dreisbach, G., \& Haider, H. (2008). That's what task sets are for: Shielding against irrelevant information. Psychological Research, 72, 355-361. doi:10.1007/s00426-007-0131-5 
FitTs, P. M., \& DeInINGER, R. L. (1954). S-R compatibility: Correspondence among paired elements within stimulus and response codes. Journal of Experimental Psychology, 48, 483-492.

FitTs, P. M., \& SEEgER, C. M. (1953). S-R compatibility: Spatial characteristics of stimulus and response codes. Journal of Experimental Psychology, 46, 199-210.

FitTs, P. M., \& Simon, C. W. (1952). Some relations between stimulus patterns and performance in a continuous dual-pursuit task. Journal of Experimental Psychology, 43, 428-436.

Floyer-Lea, A., \& Matthews, P. M. (2004). Changing brain networks for visuomotor control with increased movement automaticity. Journal of Neurophysiology, 92, 2405-2412.

Gilbert, S. J., \& Shallice, T. (2002). Task switching: A PDP model. Cognitive Psychology, 44, 297-337.

Grafton, S. T., Hazeltine, E., \& Ivry, R. B. (1998). Abstract and effector-specific representations of motor sequences identified with PET. Journal of Neuroscience, 18, 9420-9428.

Grafton, S. T., Hazeltine, E., \& Ivry, R. B. (2002). Motor sequence learning with the nondominant left hand. A PET functional imaging study. Experimental Brain Research, 146, 369-378.

HALSBAND, U. (1992). Left hemisphere preponderance in trajectorial learning. NeuroReport, 3, 397-400.

HasbroucQ, T., Burle, B., Akamatsu, M., Vidal, F., \& Possamaï, C.-A. (2001). An electromyographic investigation of the effect of stimulus-response mapping on choice reaction time. Psychophysiology, 38, 157-162.

Hazeltine, E. (2005). Response-response compatibility during bimanual movements: Evidence for the conceptual coding of action. Psychonomic Bulletin \& Review, 12, 682-688.

Hazeltine, E., Bunge, S. A., Scanlon, M. D., \& Gabrieli, J. D. E. (2003). Material-dependent and material-independent selection processes in the frontal and parietal lobes: An event-related fMRI investigation of response competition. Neuropsychologia, 41, 1208-1217.

Imamizu, H., \& Shimojo, S. (1995). The locus of visual-motor learning at the task or manipulator level: Implications from intermanual transfer. Journal of Experimental Psychology: Human Perception \& Performance, 21, 719-733.

JERSILD, A. T. (1927). Mental set and shift. Archives of Psychology, 14, 5-81.

Jost, K., MAYr, U., \& RösLER, F. (2008). Is task switching nothing but cue priming? Evidence from ERPs. Cognitive, Affective, \& Behavioral Neuroscience, $\mathbf{8}, 74-84$

Jueptner, M., Stephan, K. M., Frith, C. D., Brooks, D. J., Frackowiak, R. S. J., \& Passingham, R. E. (1997). Anatomy of motor learning: I. Frontal cortex and attention to action. Journal of Neurophysiology, 77, 1313-1324.

Kimberg, D. Y., Aguirre, G. K., \& D'Esposito, M. (2000). Modulation of task-related neural activity in task-switching: An fMRI study. Cognitive Brain Research, 10, 189-196.

Kornblum, S., \& LeE, J.-W. (1995). Stimulus-response compatibility with relevant and irrelevant stimulus dimensions that do and do not overlap with the response. Journal of Experimental Psychology: Human Perception \& Performance, 21, 855-875.

Krakauer, J. W., Pine, Z. M., Ghilardi, M. F., \& Ghez, C. (2000). Learning of visuomotor transformations for vectorial planning of reaching trajectories. Journal of Neuroscience, 120, 8916-8924.

Kveraga, K., Boucher, L., \& Hughes, H. C. (2002). Saccades operate in violation of Hick's law. Experimental Brain Research, 146, 307-314.

Lange, R. K., Braun, C., \& Godde, B. (2006). Coordinate processing during the left-to-right hand transfer investigated by EEG. Experimental Brain Research, 168, 547-556.

Lange, R. K., Godde, B., \& Braun, C. (2004). EEG correlates of coordinate processing during intermanual transfer. Experimental Brain Research, 159, 161-171.

Lien, M. C., \& Proctor, R. W. (2002). Stimulus-response compatibility and psychological refractory period effects: Implications for response selection. Psychonomic Bulletin \& Review, 9, 212-238.

LogAn, G. D., \& Bundesen, C. (2003). Clever homunculus: Is there an endogenous act of control in the explicit task-cuing procedure? Journal of Experimental Psychology: Human Perception \& Performance, 29, 575-599.
Logan, G. D., \& Bundesen, C. (2004). Very clever homunculus: Compound stimulus strategies for the explicit task-cuing procedure. Psychonomic Bulletin \& Review, 5, 832-840.

Los, S. A. (1996). On the origin of mixing costs: Exploring information processing in pure and mixed blocks of trials. Acta Psychologica, 94, $145-188$.

Malfait, N., Shiller, D. M., \& Ostry, D. J. (2002). Transfer of motor learning across arm configurations. Journal of Neuroscience, 22 , 9656-9660.

MaYr, U., \& Kliegl, R. (2003). Differential effects of cue changes and task changes on task-set selection costs. Journal of Experimental Psychology: Learning, Memory, \& Cognition, 29, 362-372.

Meiran, N. (1996). Reconfiguration of processing mode prior to task performance. Journal of Experimental Psychology: Learning, Memory, \& Cognition, 22, 1423-1442.

MeIran, N. (2000a). Modeling cognitive control in task-switching. Psychological Research, 63, 234-249.

Meiran, N. (2000b). Reconfiguration of stimulus task sets and response task sets during task switching. In S. Monsell \& J. Driver (Eds.), Control of cognitive processes: Attention and performance XVIII (pp. 377399). Cambridge, MA: MIT Press.

Miyake, A., Friedman, N. P., Emerson, M. J., Witzki, A. H., HowERTER, A., \& WAgER, T. D. (2000). The unity and diversity of executive functions and their contributions to complex "frontal lobe" tasks: A latent variable analysis. Cognitive Psychology, 41, 49-100.

Monsell, S. (2003). Task switching. Trends in Cognitive Sciences, 7, 134-140.

Morton, S. M., LANG, C. E., \& Bastian, A. J. (2001). Inter- and intralimb generalization of adaptation during catching. Experimental Brain Research, 141, 438-445.

Nefs, H. T., Kappers, A. M. L., \& KoenderinK, J. J. (2005). Intermanual and intramanual tactual grating discrimination. Experimental Brain Research, 163, 123-127.

Nelson, M. D., \& Hughes, H. C. (2007). Inhibitory processes mediate saccadic target selection. Perceptual Motor Skills, 105, 939-958.

Osman, A., Bashore, T. R., Coles, M. G., Donchin, E., \& Meyer, D. E. (1992). On the transmission of partial information: Inferences from movement-related brain potentials. Journal of Experimental Psychology: Human Perception \& Performance, 18, 217-232.

PAshler, H. (2000). Task switching and multitask performance. In S. Monsell \& J. Driver (Eds.), Control of cognitive processes: Attention and performance XVIII (pp. 277-307). Cambridge, MA: MIT Press.

Perez, M. A., Tanaka, S., Wise, S. P., Sadato, N., Tanabe, H. C., Willingham, D. T., \& Cohen, L. G. (2007). Neural substrates of intermanual transfer of a newly acquired motor skill. Current Biology, 17, 1896-1902.

Proctor, R. W., WANG, H., \& VU, K.-P. (2002). Influences of different combinations of conceptual, perceptual, and structural similarity on stimulus-response compatibility. Quarterly Journal of Experimental Psychology, 55A, 59-74.

Puttemans, V., Wenderoth, N., \& Swinnen, S. P. (2005). Changes in brain activation during the acquisition of a multifrequency bimanual coordination task: From the cognitive stage to advanced levels of automaticity. Journal of Neuroscience, 25, 4270-4278.

Rogers, R. D., \& Monsell, S. (1995). Costs of a predictable switch between simple cognitive tasks. Journal of Experimental Psychology: General, 124, 207-231.

Rubinstein, J. S., Meyer, D. E., \& Evans, J. E. (2001). Executive control of cognitive processes in task switching. Journal of Experimental Psychology: Human Perception \& Performance, 27, 763-797.

Sathian, K., \& Zangaladze, A. (1998). Perceptual learning in tactile hyperacuity: Complete intermanual transfer but limited retention. Experimental Brain Research, 118, 131-134.

Schneider, D. W., \& Logan, G. D. (2005). Modeling task switching without switching tasks: A short-term priming account of explicitly cued performance. Journal of Experimental Psychology: General, 134, 343-367.

SCHNEIDER, D. W., \& LogAN, G. D. (2007). Retrieving information from a hierarchical plan. Journal of Experimental Psychology: Learning, Memory, \& Cognition, 33, 1076-1091.

SCHULZE, K., LÜDERS, E., \& JäNCKE, L. (2002). Intermanual transfer in a simple motor task. Cortex, 38, 805-815. 
Shadmehr, R., \& Moussavi, Z. M. (2000). Spatial generalization from learning dynamic of reaching movements. Journal of Neuroscience, 20, 7807-7815

ShadmeHr, R., \& Mussa-Ivaldi, F. A. (1994). Adaptive representation of dynamics during learning of motor task. Journal of Neuroscience, 14, 3208-3224

Simon, J. R., \& Rudell, A. P. (1967). Auditory S-R compatibility: The effect of an irrelevant cue on information processing. Journal of Applied Psychology, 51, 300-304.

Simon, J. R., \& SMALL, A. M., JR. (1969). Processing auditory information: Interference from an irrelevant cue. Journal of Applied Psychology, 53, 433-435.

Stoet, G., \& SNyder, L. H. (2003). Executive control and task switching in monkeys. Neuropsychologia, 41, 1357-1364.

Stoet, G., \& Snyder, L. H. (2007). Extensive practice does not eliminate human switch costs. Cognitive, Affective, \& Behavioral Neuroscience, 7, 192-197.

TaYloR, H. G., \& Heilman, K. M. (1980). Left-hemisphere motor dominance in righthanders. Cortex, 16, 587-603.

TeIXEIRA, L. A. (2000). Timing and force components in bilateral transfer of learning. Brain \& Cognition, 44, 455-469.

Theios, J. (1975). The components of response latency in simple human information processing tasks. In P. M. A. Rabbitt \& S. Dornic (Eds.), Attention and performance $V$ (pp. 418-440). New York: Academic Press.

Thut, G., Cook, N. D., Regard, M., Leenders, K. L., Halsband, U., \& LANDIS, T. (1996). Intermanual transfer of proximal and distal motor engrams in humans. Experimental Brain Research, 108, 321-327.
Ungerleider, L. G., Doyon, J., \& KARNI, A. (2002). Imaging brain plasticity during motor skill learning. Neurobiology of Learning \& Memory, 78, 553-564.

VAnGHeluwe, S., Wenderoth, N., \& Swinnen, S. P. (2005). Learning and transfer of an ipsilateral coordination task: Evidence for a duallayer movement representation. Journal of Cognitive Neuroscience, 17, 1460-1470.

Wahl, M., Lauterbach-Soon, B., Hattingen, E., Jung, P., SingER, O., Volz, S., ET AL. (2007). Human motor corpus callosum: Topography, somatotopy, and link between microstructure and function. Journal of Neuroscience, 27, 12132-12138.

WANG, J., \& SAINBURG, R. L. (2004). Interlimb transfer of novel inertial dynamics is asymmetrical. Journal of Neurophysiology, 92, 349-360.

Willingham, D. B. (1998). A neuropsychological theory of motor skill learning. Psychological Review, 105, 558-584.

Willingham, D. B., Wells, L. A., Farrell, J. M., \& Stemwedel, M. E. (2000). Implicit motor sequence learning is represented in response locations. Memory \& Cognition, 28, 366-375.

Woodworth, R. S., \& SchlosberG, H. (1954). Experimental Psychology. New York: Holt, Rinehart \& Winston.

Wylie, G., \& Allport, A. (2000). Task switching and the measurement of "switch costs." Psychological Research, 63, 212-233.

(Manuscript received December 19, 2006; revision accepted for publication November 11,2008.) 